\title{
Computer Simulations of a New Three Rods Ion Optic (TRIPOLE) with High Focusing and Mass Filtering Capabilities
}

\author{
Gary Abdiel Salazar and Tsutomu Masujima \\ Analytical Molecular Medicine and Devices Laboratory, Graduate School of Biomedical Sciences, Hiroshima \\ University, Hiroshima, Japan
}

\begin{abstract}
A novel three rod (tripole) ion optic to which three AC voltages with symmetrically delayed phase shifts were applied to each electrode. We studied its ion guiding, focusing, and mass filtering capabilities by SIMION ver. 7.0 computer simulations. An electric field mathematical model was developed to calculate the pseudopotential of the tripole radial AC force. The tripole showed stable ion guiding for wide ranges of AC amplitude; better collisional focusing than hexapole and octapole and similar focusing as quadrupole (rod pole). Also, the ion optic clearly showed interesting mass filtering potential when the phase shift was asymmetrically delayed. The symmetric shape of the pseudopotential field explained the tripole ion guiding and focusing capabilities. For mass filtering, the pseudopotential was asymmetric and its effect was balanced with DC voltage to separate the ions, depending in their masses. The resolution was much lower than quadrupole but useful when rough filtering was required. (J Am Soc Mass Spectrom 2007, 18, 413-421) @ 2007 American Society for Mass Spectrometry
\end{abstract}

$\mathrm{I}$ on guide and ion focusing devices are essential in mass spectrometry. For example, the beam must be focused into the small differential pumping apertures for high ion transmission. The resolution and transmission of a not-well-focused ion beam through a quadrupole decreases because many ions are randomly lost due to imperfect fields located near the electrodes [1]. Similar situation of focused beam affects the resolution of the orthogonal time-of-flight mass spectrometer [2]. Mass selection is the heart of mass spectrometry and the quadrupole has governed AC voltage mass filtering for a long time, but science should continue the search for new and less expensive filters.

We are introducing an ion optic with three AC poles, hereafter named as "tripole," with better collisional focusing capabilities than hexapole and octapole. Also, the tripole showed interesting mass filtering potential.

\section{Ion Guiding and Collisional Focusing}

In $\mathrm{AC}$ ion guides the ions can be confined in radial dimension due to the presence of inhomogeneous $\mathrm{AC}$ field [3]. Under certain conditions, ion motion in any inhomogeneous AC field can be described by the pseudo or effective potential theory [3-9]. If the ion loses kinetic energy due to inelastic collisions with neutral gas particles, it will move to areas of low pseudopotential located near the center [10]. The pseu-

Published online December 4, 2006

Address reprint requests to Dr. T. Masujima, Humanix, Hiroshima University, 1-2-3 Kasumi, Minami-ku, Hiroshima City, Hiroshima 734-8551, Japan.E-mail: tsutomu@hiroshima-u.ac.jp

dopotential can be calculated and expressed as electric potential with the eq 1 (Dehmelt, H. G., equation) [11].

$$
\psi=\left(e / 4 m \omega^{2}\right) E_{0}^{2}
$$

where $m$ is the ion mass $(\mathrm{kg}), e$ is the ion charge (Coulombs), $\omega$ is the AC voltage frequency in $\mathrm{rad} / \mathrm{s}$, and $E_{0}$ is the amplitude of the AC electric field.

Collisional focusing in 2D guides has been useful since it was established [12], and its strength can be critical for capturing ions with high mass and high initial velocity distribution. For example, with a QqTOF instrument, high mass proteins have been well detected in intact form [13]. Also, MALDI time-of-flight signals for Alzheimer biomarkers have been improved [14]. Efficient ion guides with inert or reactive gas are necessary and useful in collisioninduced dissociation (CID) and inductively coupled plasma (ICP) reaction cells $[15,16]$.

\section{Experimental}

\section{Conventional and Novel AC Ion Optic}

Looking for new ion optics with simple electrode arrangements and high collisional focusing, we are introducing a new AC-ion optic-like multipole that consists of three parallel cylindrical electrodes equally aligned in radial symmetry around the central z-axis, defining an inscribed space with radius $\left(r_{0}\right)$. Three AC voltages, symmetrically delayed to phase shifts of 0,120 , and 240 degrees are applied to each electrode with the aim of creating a rotational electric field, see Figure 1. For understanding and predicting the tripole guiding, focusing, and filtering capabilities; we studied the ion 


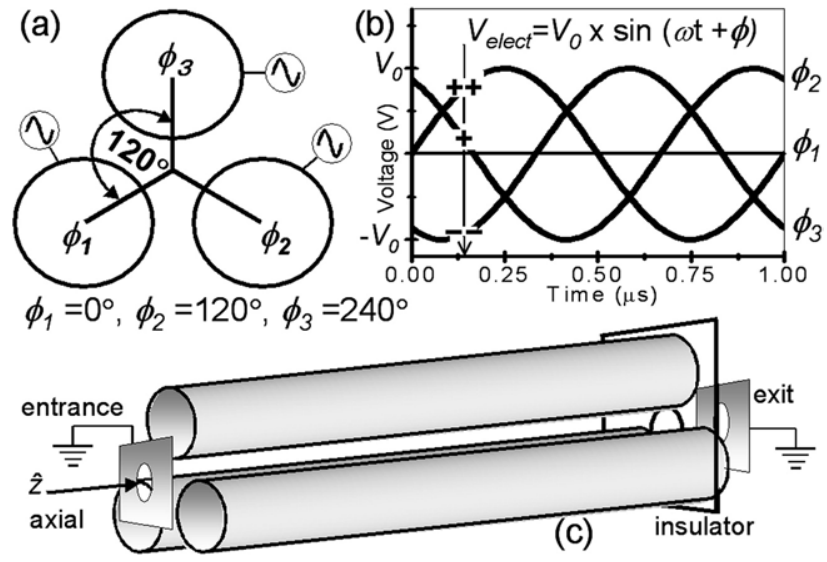

Figure 1. Tripole scheme. (a) Tripole cross-section with symmetric rod positioning; (b) three AC voltages with phase shift applied to each rod; (c) 3D view with entrance and exit metal apertures, and insulator plate. The insulator is used only when using the tripole as mass filter.

movement inside this novel arrangement by computer simulations and by calculating its pseudopotential field.

The electric potential $(V)$ in polar coordinates can be easily calculated for multipoles:

$$
\begin{aligned}
& V(r, \theta, t)=V_{0}\left(r / r_{0}\right)^{N} \cos (N \theta) \cos (\omega t) \text { and } \\
& \left|E_{0}\right|=N V_{0} / r_{0}\left(r / r_{0}\right)^{N-1}
\end{aligned}
$$

where $V_{0}$ is the zero to peak AC voltage amplitude, $r_{0}$ is the field radius, and $N$ is the number of pairs of parallel rods [17]. A mathematical model for the tripole radial electric potential and pseudopotential was developed because calculating the pseudopotential from SIMION data is an impractical and very time-consuming method that only SIMION users can reproduce. Tripole potential will be a complicated mixture of many other multipoles, each with similar amplitude.

Also, calculations of collisions with background gas were added to the simulations, to study collisional focusing and compare with conventional guides. Because the stability diagram is the essence of mass filtering, we would like to find the tripole DC-AC ion stability ranges to find a mass filtering behavior.

\section{Computational Methods}

\section{Tripole Pseudopotential Mathematical Model}

For the tripole, an electric field mathematical model was developed by fitting the electric field calculated with SIMION ver. 7.0 3D. SIMION SL TOOLS software was used to convert the SIMION data (binary format) in numerical format for the tripole 2-D array "main body," explained in "Ion Movement Simulations."

\section{Ion Guiding}

The AC voltages and ion-gas collisions were controlled by SIMION user-programming. For computer memory saving, the tripole was segmented in three "array instances" (entrance, main body, and exit). The segments were proportionally scaled by changing the software array density $(\mathrm{mm} / \mathrm{gu})$, where $\mathrm{mm}$ is millimeter and $g u$ is the software arbitrary distance unit [18]. We chose the instances array density thinking in a balance between computational time and simulation accuracy. The entrance instance was a 3D electric potential array with density of 0.04167 times $r_{0}\left(0.04167 x \quad r_{0}\right) \mathrm{mm} / \mathrm{gu}$; rods length $108 \mathrm{gu}$, the ratio of the electrodes $\left(r_{e}\right)$ with $r_{0}$ was $r_{\mathrm{e}} / r_{0}=2.2$; and an entrance plate with dimensions $(212 \times 212 \times 6) g u$, separated $10 \mathrm{gu}$ from the rods. Groups of 25 ions, radially positioned around the central axis, were flown individually from inside of a through-hole (radius $r_{0}$ ) in the entrance plate. The main body instance was a 2D array with density of 0.00833 $\mathrm{mm} / \mathrm{g} u$ times $r_{0}$, centered on the central axis. The 2D main body was projected in the z-axis as 3D planar symmetry by controlling the " $\mathrm{z}$ " parameter, until obtaining the tripole total length $(150 \mathrm{~mm})$. The exit instance of the tripole was exactly equal to the entrance instance, including an exit plate.

The conventional multipoles (quadrupole, hexapole, and octapole) simulation parameters were similar to the tripole but they were divided only in two array instances, entrance and main body. The multipoles entrance and main body array density were equal as for tripole but the proportion of the electrode radius with the inscribed space radius $\left(r_{e} / r_{0}\right)$ for hexapole was 0.5375 , for octapole 0.355 , and for quadrupole $1.130[19,20]$.

\section{Mass Filtering}

The SIMION simulation was similar as in the section "ion guiding" because the tripole mass filter scheme was almost equal to the tripole ion guide. The differences were: DC voltage was applied to electrode " 1 " and the AC voltage phase shift of electrode " 1 " and " 2 " were studied, searching for mass filtering behavior. An insulator plate (2 $\mathrm{mm}$ thick) with a through-hole of radius $\left(r_{0}\right)$ centered on the longitudinal axis was placed at the edge of the tripole end, just before the exit plate. The beam initial conditions were: groups of 25 ions were flown together with kinetic energy $10 \pm 10 \% \mathrm{eV}$, elevation angle el $= \pm 5^{\circ}$, azimuthal angle az $= \pm 90^{\circ}$, initial position $y= \pm 0.05 \mathrm{~mm}, \mathrm{x}= \pm 0.05 \mathrm{~mm}$ from the center, and the initial time-of-birth delay was $0<$ TOB $<0.9 \mu$ s. Trying to simulate real ion beams, the initial conditions were randomized using the algorithm included in SIMION package demonstrations.

\section{Theory and Calculation}

\section{Collisional Focusing Model}

We chose the ion/neutral hard sphere collision model developed by Dahl, D. [21]. In our work, the gas particle speed was fixed at the root mean square of the Maxwell-Boltzmann distribution $\left(\mathrm{V}_{\mathrm{rms}}\right)$ instead of ran- 


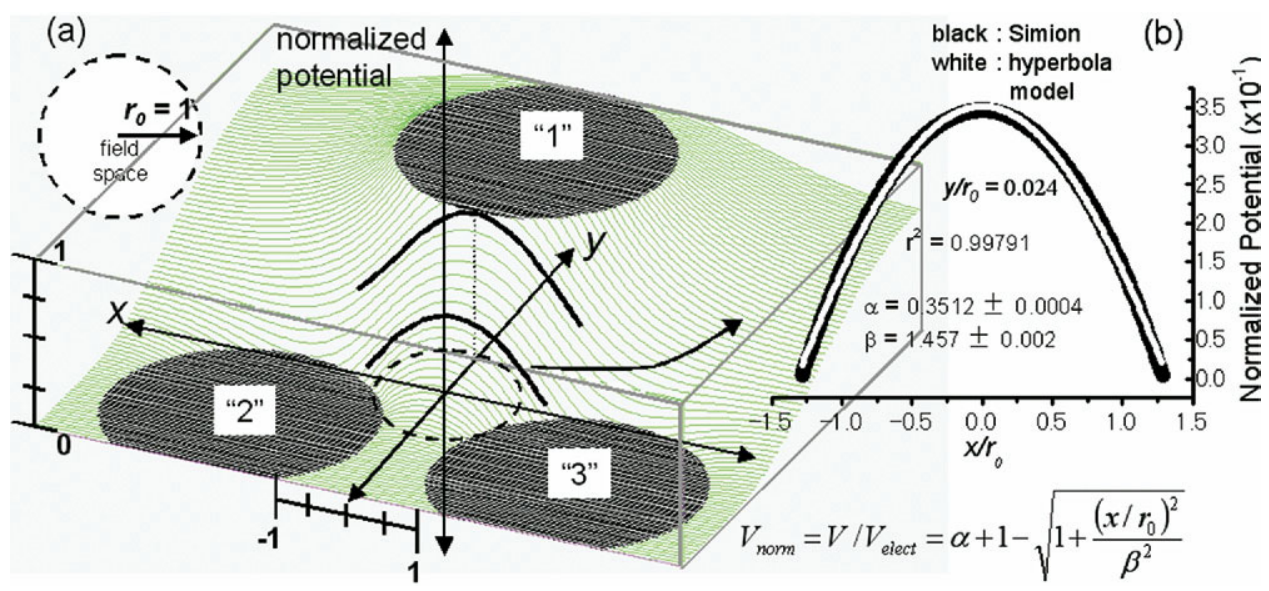

Figure 2. Tripole electric potential surface in cartesian coordinates calculated by SIMION. (a) The potential has been normalized to the potential of the electrode " 1 " and the cartesian coordinates has been normalized to the field radius $\left(r_{0}\right) ;(\mathbf{b})$ hyperbolic shape of the normalized potential at a fixed $y / r_{0}$ position, highly fitted with the proposed mathematical model.

domly distributing it. This model includes approaches that simulate real systems such as thermal broadening, scattering angle, "rear-end," and "head-on" elastic collisions. The ion-gas collision cross-sections were approximated by $\sigma=K \times m^{b}$ [22]. The constants $K$ and $b, 2.41 \AA^{2}$ and 0.664 , respectively, were obtained by fitting the cross section values from the mass dependent database of Valentine et al. [23], and ion mass " $m$ " is in Daltons.

\section{Tripole Normalized Electric Potential Curve and Electric Field}

By numerical analysis of the electric potential, calculated by SIMION, we developed and fitted a mathematical model. Figure 2 represents the electric potential surface of tripole cross-section with electrode field radius ratio $\left(r_{e} / r_{0}\right)$ of 2.2, with positive voltage applied to the electrode " 1, " and the others are grounded. The $x$-y Cartesian coordinates were normalized over $r_{0}\left(x / r_{0}\right.$, $\left.y / r_{0}\right)$ and the electric potential values were normalized over the voltage of electrode "1" $\left(V 1 / V 1_{\text {elect }}\right)$.

\section{Numerical Analysis of the Normalized Potential Curve}

Let us approach the curve of normalized electric potential versus $x / r_{0}$ position, at any determined $y / r_{0}$ position inside the field space (see Figure 2), with a modified hyperbola equation written as eq 3 . The hyperbola canonical equation was modified by fixing the root to negative and by adding the factor $(\alpha+1)$. These modifications fix the vertex in the $V-y$ plane and its direction will depend on the electrode voltage polarity, $\alpha$ will be equal to the normalized potential at the vertex, and $\beta$ will be proportional to the hyperbola width.

$$
V_{\eta}=V / V_{\text {elect }}=\alpha+1-\sqrt{1+\frac{\left(x / r_{0}\right)^{2}}{\beta^{2}}}
$$

Eq 3 was applied to 25 hyperbolas, located at different $y / r_{0}$ positions from -1 to 1 , for the tripole electric field $\left(r_{0}=3 \mathrm{~mm}, V 1_{\text {elect }}=100 \mathrm{~V}\right)$. One typical example of the eq 3 fitting is shown in Figure $2 b$ and the average
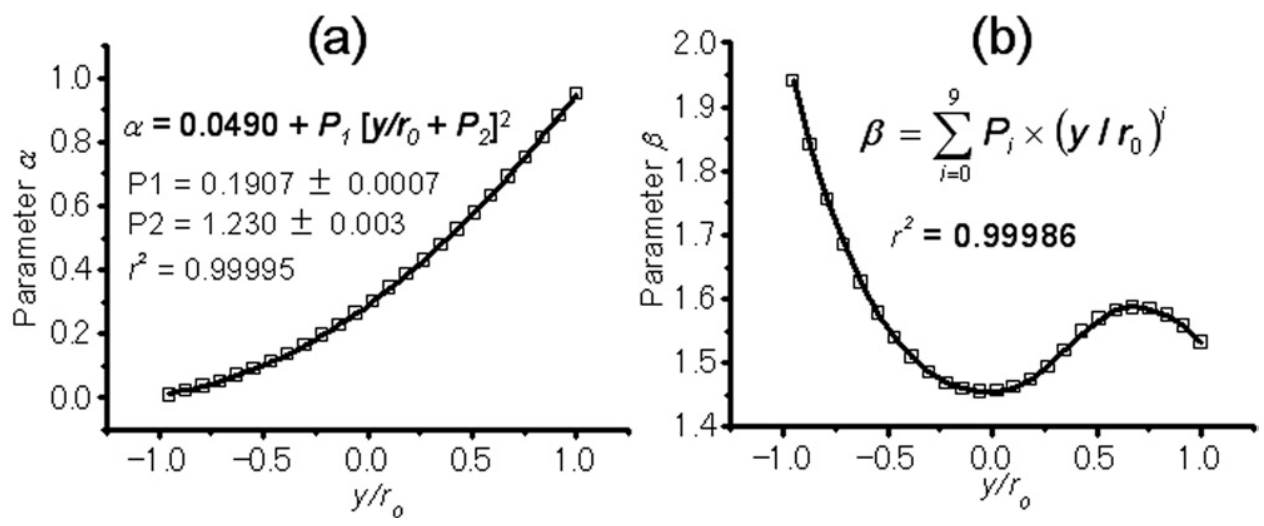

Figure 3. Hyperbola model parameters for tripole electric field, $n=25$. (a) $\alpha$ Parameter with quadratic behavior; (b) $\beta$ parameters with polynomial behavior. 
Table 1. Dimensionless $\beta$ parameters obtained for equation $11^{\mathrm{a}}$

\begin{tabular}{lcl}
\hline & Equation $\beta$ parameters & $\begin{array}{c}\text { Std.dev. } \\
(\sigma)\end{array}$ \\
\hline \hline$P_{0}$ & 1.45351 & 0.0009 \\
$P_{1}$ & 0.02064 & 0.006 \\
$P_{2}$ & 0.49666 & 0.02 \\
$P_{3}$ & 0.24903 & 0.07 \\
$P_{4}$ & -0.31775 & 0.09 \\
$P_{5}$ & -1.55653 & 0.2 \\
$P_{6}$ & 0.08174 & 0.1 \\
$P_{7}$ & 1.75208 & 0.3 \\
$P_{8}$ & 0.06235 & 0.09 \\
$P_{9}$ & -0.70992 & 0.2 \\
\hline
\end{tabular}

${ }^{\text {a }}$ The values were obtained by iterative minimization of the squared deviation.

bStandard deviation of the parameter value.

variance coefficient $\left(r^{2}\right)$ was 0.9988 with a confidence interval \pm 0.0004 (confidence $99.0 \%$ ); therefore, we can say that the hyperbolic model fits the SIMION electric potential data very well.

The parameters $\alpha$ and $\beta$ are plotted in Figure 3, and we can clearly observe that $\alpha$ is best described by a quadratic function $\alpha=J_{0}+J_{1}\left[y / r_{0}+J_{2}\right]^{2}$, eq 4 , where $J_{0}=0.0490, J_{1}=0.1907$, and $J_{2}=1.230 . \beta$ is described with a polynomial function of the position

$$
\beta=\sum_{n=0}^{9} P_{n} \times\left(y / r_{0}\right)^{n}
$$

$y / r_{0}$, and the respective parameters are listed in table 1. The parameters of eq 5 were reported with 5 decimals because high-order polynomials are exponentially sensitive to small variations of the parameters significant digits that lead to considerable interpolation deviations. Using the parameters as indicated in Table 1, the sum of the squared deviations is $5 \times 10^{-5}$. But if we use the significant digits, indicated by the standard deviation of each parameter then the sum of the squared deviations of Figure $3 \mathrm{~b}$ jumps to $1 \times 10^{-3}$. The high polynomial was reported because the correlation improved until ninth order, lower was not satisfactory. High order functions are common in analytical and numerical solutions of electric potential, for example, the Kingdon trap electric potential is represented with an infinite series of cosines [24]. Finally, the equations are rewritten in polar coordinates:

$$
\begin{aligned}
& V_{\eta}(r, \theta)=V / V_{\text {elect }}=\alpha+1-\sqrt{1+\frac{\left(r / r_{0} \cos \theta\right)^{2}}{\beta^{2}}} \\
& \alpha(r, \theta)=J_{0}+J_{1}\left[r / r_{0} \sin \theta+J_{2}\right]^{2} \\
& \beta(r, \theta)=\sum_{n=0}^{9} P_{n} \times\left(r / r_{0} \sin \theta\right)^{\mathrm{n}}
\end{aligned}
$$

When the eqs 7 and 8 are used in eq 6, the solution of the normalized potential is precisely obtained for electrode " 1 ".

By symmetry, the other two electrodes have the same normalized potential if the angle coordinate value is rotated $120^{\circ}$ from electrode 1 to electrode 2 and $-120^{\circ}$ to electrode 3. Thus, $\Theta_{1}=\theta$ for electrode " 1 ", $\Theta_{2}=\theta-$ $2 \pi / 3 \mathrm{rad}$ for electrode " 2 ", and $\Theta_{3}=\theta+2 \pi / 3 \mathrm{rad}$ for electrode " 3 " where $\theta$ is the angle coordinate relative to electrode " 1 ". The composite potential curve can be obtained by simple arithmetical addition of the contribution of all the electrodes (superposition principle) $V(r, \Theta)=V 1(r, \Theta)+V 2(r, \Theta)+V 3(r, \Theta)$, eq 9. Placing eq 6 , with the rotation transformations, in eq 9 leads to $V(r, \Theta)=\left[V 1_{\text {elect }} \times V 1 \eta\left(r, \Theta_{1}\right)\right]+\left[V 2_{\text {elect }} \times V 2 \eta\left(r, \Theta_{2}\right)\right]+$ $\left[V 3_{\text {elect }} \times V 3_{\eta}\left(r, \Theta_{3}\right)\right.$ eq 10 , where $V_{\text {elect }}$ is the electrode time-dependent voltage and $V i_{\eta}$ is the normalized potential. The negative gradient of eq 10 leads to the electric field components:

$$
\begin{aligned}
E_{r}= & -\left[V_{0} \sin (\omega t) \times \dot{V} 1_{\eta r}\right]-\left[V_{0} \sin \left(\omega t+\phi_{2}\right) \times \dot{V} 2_{\eta r}\right] \\
& -\left[V_{0} \sin \left(\omega t+\phi_{3}\right) \times \dot{V} 3_{\eta r}\right]
\end{aligned}
$$

where $\dot{V} i_{\eta r}=\frac{\partial V i_{\eta}}{\partial r}$ for electrode " $\mathrm{i}$ ".

$$
\begin{aligned}
E_{\Theta}= & -\left[V_{0} \sin (\omega t) \times \dot{V} 1_{\eta \Theta}\right]-\left[V_{0} \sin \left(\omega t+\phi_{2}\right) \times \dot{V} 2_{\eta \Theta}\right] \\
& -\left[V_{0} \sin \left(\omega t+\phi_{3}\right) \times \dot{V} 3_{\eta \Theta}\right]
\end{aligned}
$$

$$
\dot{V} i_{\eta \Theta}=\frac{1}{r} \frac{\partial i_{\eta}}{\partial \Theta} \text { for electrode “i." }
$$

Using eqs 11 and 12, to solve the algebraic procedure $|E|=\sqrt{\left[\left(E_{r}\right)^{2}+\left(E_{\Theta}\right)^{2}\right]}$, we can write the total electric field as:

$$
\begin{aligned}
& |E(r, \Theta, t, \phi)| \\
& =V_{0} \sqrt{\left[A \sin ^{2}(\omega t)+B \sin (\omega t) \cos (\omega t)+C \cos ^{2}(\omega t)\right]}
\end{aligned}
$$

where

$$
\begin{aligned}
& A(r, \Theta, \phi)=\dot{V} 1_{\eta \mathrm{r}}^{2}+\dot{V} 1_{\eta \Theta}^{2}+2 \cos \left(\phi_{2}\right)\left(\dot{V} 1_{\eta r} \dot{V} 2_{\eta r}\right. \\
& \left.\quad+\dot{V} 1_{\eta \Theta} \dot{V} 2_{\eta \Theta}\right)+2 \cos \left(\phi_{3}\right)\left(\dot{V} 1_{\eta r} \dot{V} 3_{\eta r}+\dot{V} 1_{\eta \Theta} \dot{V} 3_{\eta \Theta}\right) \\
& \quad+2 \cos \left(\phi_{2}\right) \cos \left(\phi_{3}\right)\left(\dot{V} 2_{\eta r} \dot{V} 3_{\eta r}+\dot{V} 2_{\eta \Theta} \dot{V} 3_{\eta \Theta}\right) \\
& \quad+\cos ^{2}\left(\phi_{2}\right)\left(\dot{V} 2_{\eta \mathrm{r}}^{2}+\dot{V} 2_{\eta \Theta}^{2}\right)+\cos ^{2}\left(\phi_{3}\right)\left(\dot{V} 3_{\eta \mathrm{r}}^{2}+\dot{V} 3_{\eta \Theta}^{2}\right)
\end{aligned}
$$




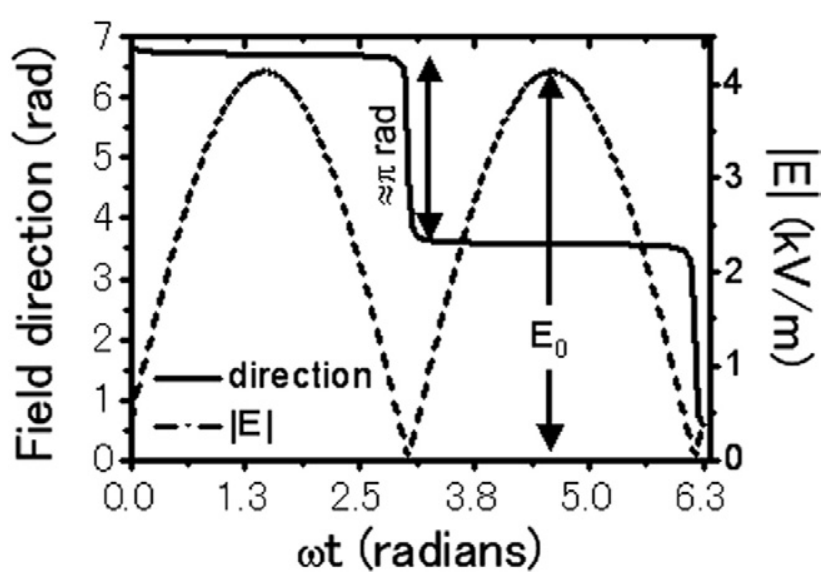

Figure 4. Typical tripole radial electric field showing a sinusoidal shape and its direction periodically becomes opposite. $E_{0}$ is the maximum field in the AC field period.

$$
\begin{aligned}
& B(r, \Theta, \phi)=2 \sin \left(\phi_{2}\right)\left(\dot{V} 1_{\eta r} \dot{V} 2_{\eta r}+\dot{V} 1_{\eta \Theta} \dot{V} 2_{\eta \Theta}\right) \\
& +2 \sin \left(\phi_{3}\right)\left(\dot{V} 1_{\eta r} \dot{V} 3_{\eta r}+\dot{V} 1_{\eta \Theta} \dot{V} 2_{\eta \Theta}\right) \\
& +2 \cos \left(\phi_{2}\right) \sin \left(\phi_{2}\right)\left(\dot{V} 2_{\eta \mathrm{r}}^{2}+\dot{V} 2_{\eta \Theta}^{2}\right)+2\left[\cos \left(\phi_{2}\right) \sin \left(\phi_{3}\right)\right. \\
& \left.+\cos \left(\phi_{3}\right) \sin \left(\phi_{2}\right)\right]\left(\dot{V} 2_{\eta r} \dot{V} 3_{\eta r}+\dot{V} 2_{\eta \Theta} \dot{V} 3_{\eta \Theta}\right) \\
& +2 \cos \left(\phi_{3}\right) \sin \left(\phi_{3}\right)\left(\dot{V} 3_{\eta \mathrm{r}}^{2}+\dot{V} 3_{\eta \Theta}^{2}\right) \\
& C(r, \Theta, \phi)=2 \sin \left(\phi_{2}\right) \sin \left(\phi_{3}\right)\left(\dot{V} 2_{\eta r} \dot{V} 3_{\eta r}\right. \\
& \left.+\dot{V} 2_{\eta \Theta} \dot{V} 3_{\eta \Theta}\right)+\sin ^{2}\left(\phi_{2}\right)\left(\dot{V} 2_{\eta \mathrm{r}}^{2}+\dot{V} 2_{\eta \Theta}^{2}\right) \\
& +\sin ^{2}\left(\phi_{3}\right)\left(\dot{V} 3_{\eta \mathrm{r}}^{2}+\dot{V} 3_{\eta \Theta}^{2}\right)
\end{aligned}
$$

\section{Calculation of the Tripole Pseudopotential}

Figure 4 is the typical plot of the tripole time-dependent electric field at certain $r, \Theta$ position (eq 13), which consists of a phase shifted oscillating field with frequency " $\omega$ " and the direction of the field periodically shifts to opposite. As a consequence, the ion also oscillates in a combination of a fast micromotion and a slow macromotion shown in Figure 6. These observations allowed us to apply the pseudopotential theory. The field amplitude $\left(E_{0}\right)$ was numerically calculated and placed in eq 1 to calculate the tripole pseudopotential $\Psi(r, \Theta)$ while for multipoles, eq 2 was placed in eq 1 and the results are shown in Figure 5.

We observed that the ions coming from the entrance plate and flying along the axis lost kinetic energy (KE) after entering in the tripole or, in the case of higher voltage, they were reflected back at the edge of the tripole entrance. We reasoned that the average effect of the axial field should not be zero. We could not develop a calculation for the axial pseudopotential, instead our calculation works for the axial position far from the entrance, where the axial field is zero. Figure 1 shows that the existence of the axial potential is not surprising because the $\mathrm{AC}$ voltage in any pole always becomes intermediate (marked with + ) with the other two poles
$(++)$ and $(-)$. This AC voltage and the tripole symmetry never neutralize the electric field in the tripole center, while the conventional multipoles symmetry and polarized AC voltages neutralize the electric field in the center. This axial potential may cause difficulties injecting ions with low energies or energy spreads, in comparison with other multipoles; nevertheless, the axial potential should be good for ion focusing because it is easier to focus ions, the kinetic energies of which have been decreased by the axial potential.

The radial pseudopotential well depth for tripole is 9.5e-3 V while for quadrupole is $2.4 \mathrm{e}-2 \mathrm{~V}$, hexapole $5.6 \mathrm{e}-2 \mathrm{~V}$, and octapole 1.0e-1 V. The well depth of the multipoles compared with tripole is $2.5,5.9$, and 10 greater, respectively, so the confining capability is stronger in conventional multipoles than in tripole.

In brief, a fast and reliable method to calculate tripole radial electric field and pseudopotential was successfully developed. Tripole and quadrupole pseudopotential share some similarity in their radial distribution, density, and penetration. The pseudopotential field shows the tripole capacity to keep the ions inside the field space with stable oscillations.

\section{Results and Discussion}

\section{Comparison Between the Ion Guiding of the Tripole and the Conventional Multipoles Without Background Gas}

Using a tripole with $r_{0}=3 \mathrm{~mm}$, the $r_{e} / r_{0}$ ratio $=2.2$ optimized the ion stable transmission. The ratio $=2.2$ was not critical because different ratios slightly decreased the ion transmission. Wide ranges of AC voltages can be applied to the tripole to get stable ion trajectories (Figure 6a-c) for ion masses from 500 to $4000 \mathrm{Da}$, and we called these regions "AC-mass stability diagrams", Figure 6a. The conventional multipoles have wider stability diagrams (blank area); however, the tripole shows a wide stability diagram useful for efficient ion guiding. The tripole stability boundaries changed with the field radius and beam conditions (data not shown). For this reason, it is not possible to state that tripole boundary will always be narrower or wider than the multipoles. This stability boundary dependence with the initial conditions shows that tripole ion guiding is very similar to the high order multipoles (hexapole and octapole), the stability diagrams of which are not defined because $x$ and $y$ motion are strongly coupled [15].

In brief, the tripole stability limits are diffuse but wide and useful for ion guiding. Thus, the tripole ion guiding performance is satisfactory for high and low masses. From an ion guiding point of view, tripole belongs to high order multipoles because of the undefined ion stability. 

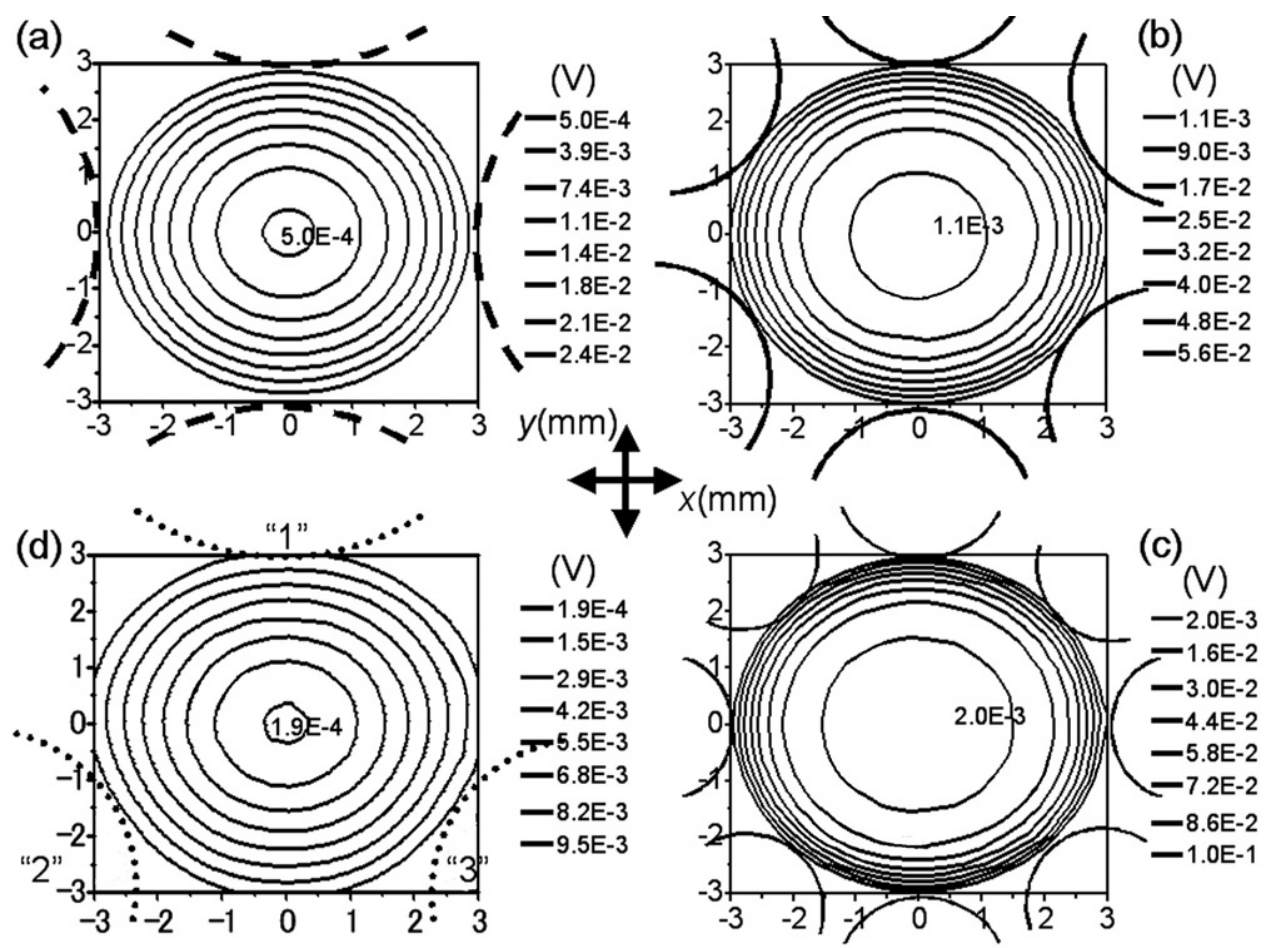

Figure 5. Radial pseudopotential contour in volts for the multipoles and tripole cross-section. All graphs show that the minimum point is in the center of each multipole and increases when it moves to the electrodes. Calculation conditions: $V_{0}=10 \mathrm{~V}, \Omega=1 \mathrm{MHz}, r_{0}=3 \mathrm{~mm}, 1000 \mathrm{Da}$, ion charge $=$ +1. (a) quadrupole; (b) hexapole; (c) octapole; (d) tripole.
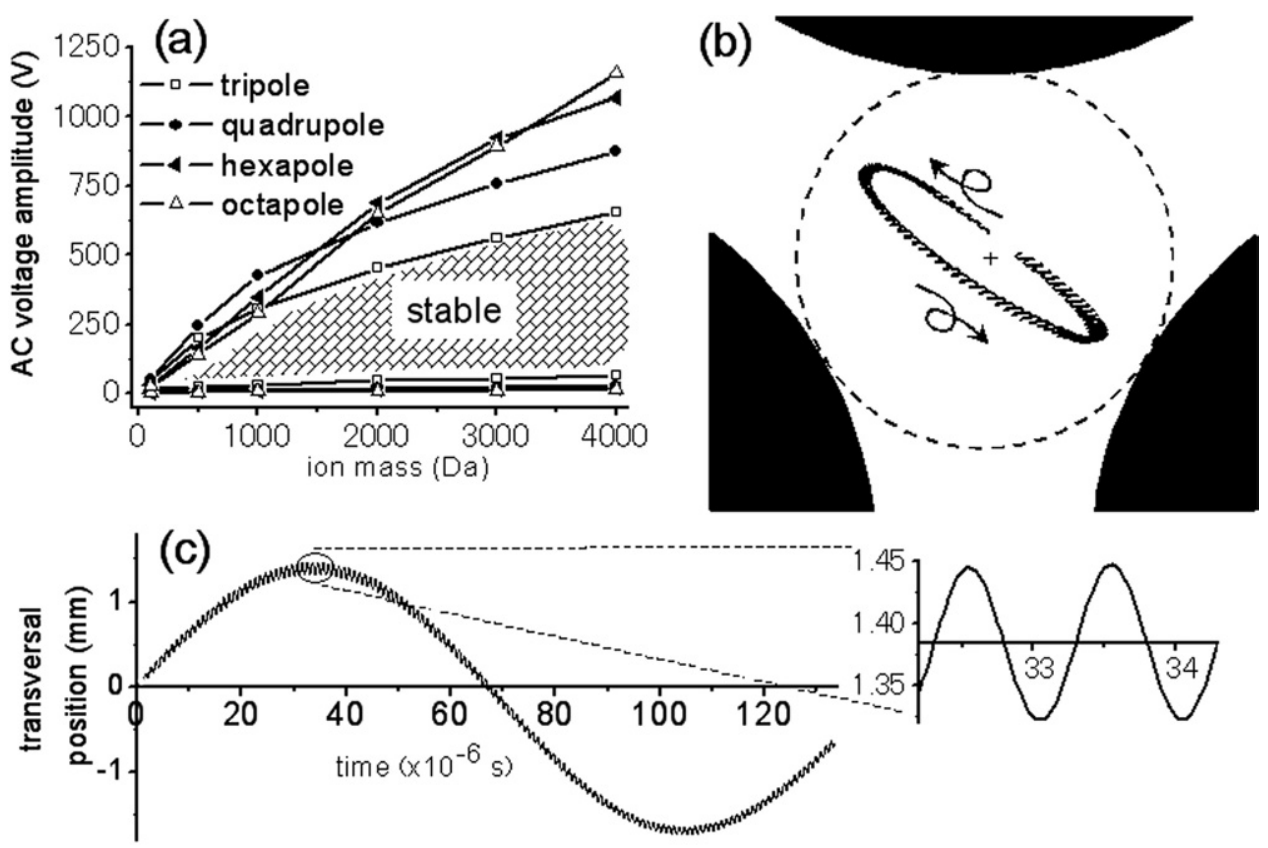

Figure 6. Ion guides $\mathrm{AC}$ voltage for stable ion guiding and tripole stable trajectory. $1 \mathrm{MHz}$, length $150 \mathrm{~mm}, r_{0}=3 \mathrm{~mm}$. Beam initial conditions: kinetic energy $10 \mathrm{eV}$, expansion angle $3^{\circ}$, and initial radius $=0.5 \times r_{0}$. (a) AC-mass stability diagrams; (b) cross-section view; (c) transversal view of the ion stable movement inside of the tripole rotational electric field. 


\section{Comparison Between the Collisional Focusing of the Tripole and Conventional Multipoles}

The focused beam radius was taken as the average of the distance from the central axis to each ion maximum oscillation, just before the device end. The tripole focused beam radius was much smaller than the hexapole and octapole and similar to the quadrupole when the initial momentum was equal to $2.30 \times 10^{-21} \mathrm{~kg} \mathrm{~m} / \mathrm{s}$ (Figure 7a and b). The AC voltages in Figure 7a and b were optimized for quadrupole and tripole as shown in Figure 7c, while hexapole and octapole focusing almost did not improve with the $\mathrm{AC}$ voltage due to the high initial kinetic energy, low gas pressure, and short multipole length. The range of mass between 1000 to 2000 Da was better focused with a certain voltage while the range between 3000 to 5000 Da focused well with a higher voltage. It was not possible to focus the whole mass range with a single high voltage because the AC field was too strong to keep stable low masses; also it is not recommendable to use high field because of the danger of collision over-heating for low masses.

The reason of tripole and quadrupole focusing superiority lies in their pseudopotential, which gradually falls to a minimum located in the center and contains the ions in regions of low radius, while not for hexapole and octapole, Figure 5. The quadrupole pseudopotential is steeper than that of the tripole, but the tripole compensates this with axial pseudopotential, which damps the ion kinetic energy and the beam becomes easier to guide and focus.

In brief, the quadrupole and tripole offer better collisional focusing than hexapoles and octapoles. The tripole or quadrupole are preferred for applications where the smallest possible focused beam diameter is required. Hexapoles and octapoles may be preferred where it is desirable to keep the electrical fields near the center as low as possible, to avoid AC heating. Quadrupole should have higher $\mathrm{AC}$ heating than tripole because quadrupole pseudopotential depth is higher.

\section{Tripole Mass Selective Electric Field and DC-AC Stability Ranges}

The mass selective electric field in the tripole could be accomplished when the AC phase shift of electrode " 3 " (Figure 1) was changed from the symmetrical $120^{\circ}$ to the asymmetrical $20^{\circ}$ and by adding DC voltage to electrode " 1 ". Tripole presented voltages ranges of DC and $\mathrm{AC}$ voltages that transmit ion masses for the given initial conditions, analogous to the quadrupole theoretical stability diagram when it is defined in voltage terms [25]. The shape of the diagram was also triangular with the narrowest transmission at the top or apex and the peak of ion transmission versus AC amplitude extracted from the apex. The height of the peak was taken to $80 \%$ and its width was measured at $25 \%$ of the peak height, which is a stricter definition than the conventional full-width at half-maximum.
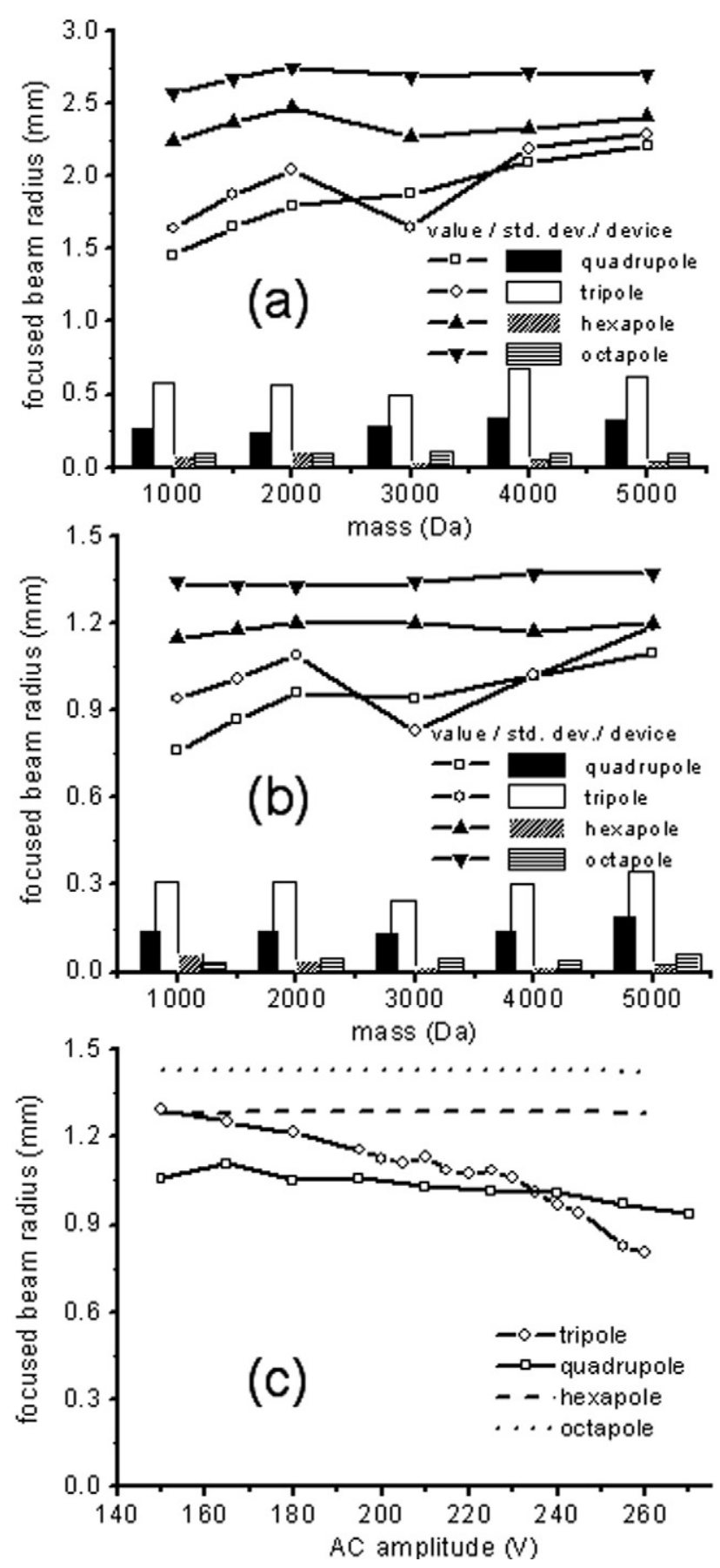

Figure 7. Focused beam radius. $3 \mathrm{mTorr} \mathrm{N}_{2}$ gas, $1 \mathrm{MHz}$, multipole length $150 \mathrm{~mm}$; initial conditions: groups of 100 ions, momentum $=2.30 \times 10^{-21} \mathrm{~kg} \mathrm{~m} / \mathrm{s}$, beam radius $=0.5 \times r_{0}$. (a) $r_{0}=6$ $\mathrm{mm}, V_{0}=510 \mathrm{~V}$ for masses 5000 to $3000 \mathrm{Da}, V_{0}=370 \mathrm{~V}$ for masses 2000 to $1000 \mathrm{Da}$; (b) $r_{0}=3 \mathrm{~mm}, V_{0}=255 \mathrm{~V}$ for masses 5000 to 3000 $\mathrm{Da}, V_{0}=175 \mathrm{~V}$ for masses 2000 to $1000 \mathrm{Da}$; (c) typical minimization of the beam radius by AC voltage in the case of $r_{0}=3 \mathrm{~mm}$ and mass $3000 \mathrm{Da}$. Tripole and quadrupole presented more focused beam than the other multipoles.

The mass resolution was calculated as the ratio between the AC voltage amplitude divided by the peak width, in voltage $\left(\mathrm{R}=\right.$ mass $/ \Delta$ mass $\left.=V_{0}^{\text {apex }} / \Delta V_{0}\right)$. The resolution in the tripole mass filter was not higher than 20 for the range of conditions: $r_{0}$ ( 3 to $6 \mathrm{~mm}$ ), AC 


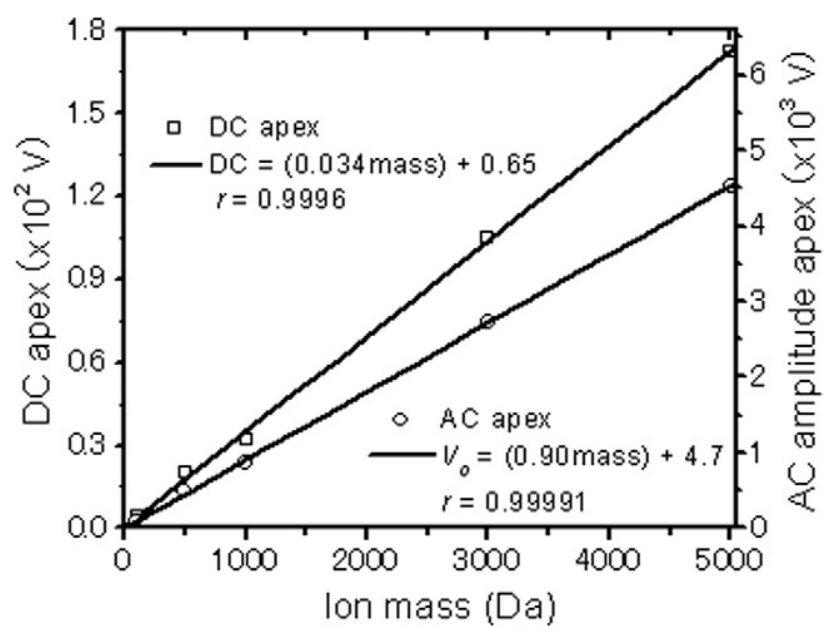

Figure 8. Linear relationship of the ion mass with the DC $(U)$ and AC $\left(V_{0-p}\right)$ amplitude voltages at the stability diagram apex. Mass selection and scanning can be done by controlling the electric conditions. $\varphi_{1}=240^{\circ}, \varphi_{2}=0^{\circ}$, and $\varphi_{3}=20^{\circ}, r_{0}=6 \mathrm{~mm}, \Omega=1$ $\mathrm{MHz}$, ion charge $=+1$.

frequency (700 to $1000 \mathrm{kHz}$ ), ion mass (500 to $3000 \mathrm{Da}$ ). The resolution of the quadrupole mass filter was much higher; nevertheless, the tripole presented clear mass filtering behavior. The ion stability in the tripole should have an ion positional dependence similar as in hexapoles and octapoles [26] affecting the AC field mass selection, and for this reason, every transmission peak presented tails. Further studies should be done to explain the presence of the tail at AC voltage higher than the peak center. Another important aspect of mass filtering is that the DC and AC voltages at the top of the stability diagram (mass selective voltages) must have a relationship with the mass, to precisely scan and select the ions. As seen in Figure 8, tripole fulfilled this need.

\section{Tripole Mass Selective Pseudopotential and Filtering Mechanism}

The Figure 9a shows the pseudopotential, calculated with the developed mathematical model (eq 13) when tripole uses only AC voltage and the electrode " 3 " phase shift is $20^{\circ}$ (asymmetric phase $\varphi_{2}=0 \mathrm{rad}, \varphi_{3}=$ $\left.1 / 9 \pi \mathrm{rad}, \varphi_{1}=4 / 3 \pi \mathrm{rad}\right)$. The model is not fulfilling boundaries conditions because some lines are orthogonal to the electrodes, and for this reason the pseudopotential is restringed to the field space. Thus, Figure $9 \mathrm{a}$ data is a more qualitative than an exact result.

The values of minimum pseudopotential are localized between electrodes " 2 " and " 3 ," and the pseudopotential increases when it moves to electrode "1." When the phase shift between electrodes " 2 " and " 3 " is close $\left(0^{\circ}\right.$ and $\left.20^{\circ}\right)$, their voltage difference is always low, while the phase shift of electrode " 1 " $\left(240^{\circ}\right)$ is well separated from the other two electrodes. This situation creates almost a dipole field with a very little rotational degree that, in overall effect, pushes the ions downward where the field is weak. If negative DC voltage is applied to the electrode " 1 ," in the case of positive ions, a constant force attracts the ions toward electrode "1." The ion trajectory is stable depending on the ion mass, $\mathrm{DC}^{\text {apex }}$ and $V_{0}{ }^{\text {apex }}$ values. This is simply Newton's law, doubling the mass and doubling the force (or voltage) gives the same equation of motion and the same transmission.

Figure $9 \mathrm{~b}$ illustrates the ion trajectories when the DC voltage is fixed at the apex and $V_{0}$ is changed for the same mass. The holed-insulator, located at the end of the tripole, avoids the ions with unstable trajectories to continue to the next ion optic, similar to the function of a sector mass spectrometer exit slit. Even the insulator introduces a charging problem; it must remain inside of the tripole mass selecting field. Outside of the tripole system the deflecting field is weak and the metal exit plate focuses all the ions, independently of their masses.

\section{Conclusions}

The tripole shares some properties with the quadrupole and high order multipoles. The tripole pseudopotential contour was symmetrical when symmetrical phase shifts were applied. The tripole pseudopotential penetrates similarly as the quadrupole pseudopotential. The
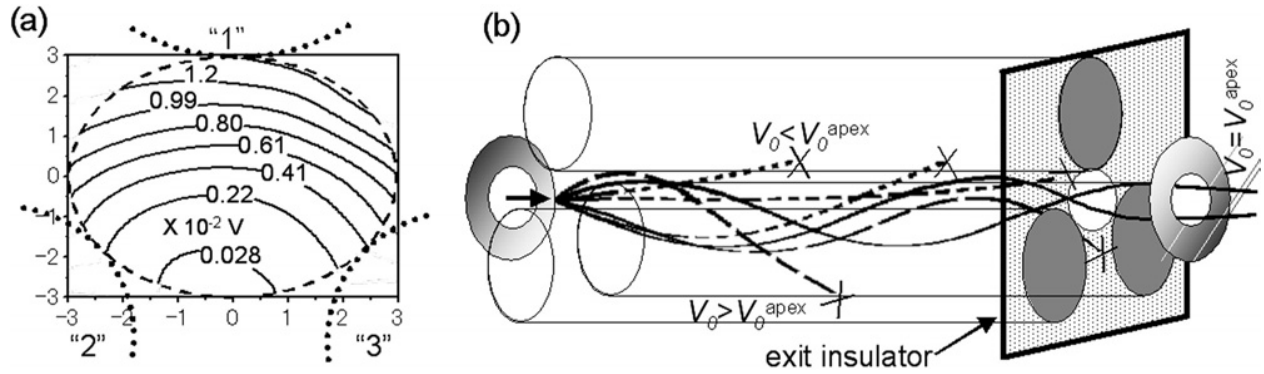

Figure 9. (a) Tripole mass filter pseudopotential contour in the field space. phase shift $\varphi_{1}=240^{\circ}, \varphi_{2}$ $=0^{\circ}$, and $\varphi_{3}=20^{\circ}, r_{0}=3 \mathrm{~mm}, 1000 \mathrm{Da}, \mathrm{AC} 1 \mathrm{MHz}, V_{0}=10 \mathrm{~V}$. The region of minimum pseudopotential is below the center. (b) SIMION simulated trajectories for tripole ion filtering: (dotted line) the AC voltage is lower than the voltage at the apex of the stability diagram; (solid line) the AC voltage is equal to the voltage at the apex of the stability diagram; (broken line) the AC voltage is higher than the voltage at the apex of the stability diagram. 
tripole AC voltage ranges were wide and useful for stably guiding high range of masses, comparable to other multipoles. Quadrupole and tripole offer better collisional focusing than high order multipoles (hexapole and octapole) because the pseudopotential can contain the ions near the center.

A mass selective electric field was created in the tripole by applying DC voltage to one electrode and changing the AC phase shift from the symmetrical $120^{\circ}$ to the asymmetrical $20^{\circ}$. The ion mass selective mechanism consisted of a balance between the DC voltage with the pseudopotential (AC average effect) acting in contrary directions. The mass selective $\mathrm{DC}$ and $\mathrm{AC}$ voltages had linear relationship with the mass, thus mass scanning was possible. The resolution in the tripole mass filter was much lower than for the rod pole quadrupole mass filter; nevertheless the tripole presented clear mass filtering behavior and is useful for rough mass filtering. This work opens a new field for investigation: multipoles with $(2 n+1)$ odd numbers of rods and with the AC applied with phase shifts of $2 \pi /(2 n+1)$.

\section{Acknowledgments}

This research was partially supported by the Japan Ministry of Education, Science, Sports, and Culture Grant-in-Aid for Scientific Research.

\section{References}

1. Blaum, K.; Gepperta, C.; Muller, P.; Nortershauser, W.; Wendt, K.; Bushaw, B. A. Peak Shape for a Quadrupole Mass Spectrometer: Comparison of Computer Simulation and Experiment. Int. J. Mass Spectrom. 2000, 202, 81-89.

2. Guilhaus, M.; Selby, D.; Mlynski, V. Orthogonal acceleration time-offlight mass spectrometry. Mass Spectrom. Rev. 2000, 19, 65-107.

3. Herbert, G.; Johnstone, R. Mass Spectrometry Basics. CRC Press LLC: Boca Raton, Florida, 2003; pp 371-382.

4. Kapitsa, P. L.; Zh. Eksperim. I Teor. Fiz. 1951, 21, 588.

5. Tolmachev, A.; Kim, T.; Udseth, H. Simulation-Based Optimization of the Electrodynamic Ion Funnel for High Sensitivity Electrospray Ionization Mass Spectrometry. Int. J. Mass Spectrom. 2000, 203, 31-47.
6. Dehmelt, H. Yu, N. Paul-Straubel-Kingdon Trap for True Zero-Point Confinement of an Individual Ion and Reservoir. Proc. Natl. Acad. Sci. U.S.A. 1997, 94, 10031-10033.

7. Armentrout, P. 2003; Theory and Ion Chemistry. Gross, M.; Caprioli R. Eds.; In The Encyclopedia of Mass Spectrometry; pp 181-195. Elsevier: Amsterdam,

8. Peik, E. Electrodynamic trap for neutral atoms. Eur. Phys. J. D.61791998183

9. Douglas, D. J.; Frank, A.J.; Mao, D. Linear Ion Traps in Mass Spectrometry. Mass Spectrom. Rev. 2005, 24, 1-29.

10. Tolmachev, A. V.; Udseth, H. R.; Smith, R. D. Modeling the Ion Density Distribution in Collisional Cooling RF Multipole Ion Guides. Int. J. Mass Spectrom. 2003, 222, 155-174.

11. Dehmelt, H. G. Radiofrequency Spectroscopy of Stored Ions. I. Storage. Adv. At. Mol. Phys. 1967, 3, 53-72.

12. Douglas, D. J.; French, J. B. Collisional Focusing Effects in Radio Frequency Quadrupoles. J. Am. Soc. Mass Spectrom. 1992, 3, 398-408.

13. Chernushevich, I. V.; Thomson, B. A. Collisional Cooling of Large Ions in Electrospray Mass Spectrometry. Anal. Chem. 2004, 76, 1754-1760.

14. Lopez, M. F.; Mikulskis, A.; Kuzdzal, S.; Bennett, D. A.; Kelly, J.; Golenko, E.; Dicesare, J.; Denoyer, E.; Patton, W. F.; Ediger, R.; Sapp, L.; Ziegert, T.; Lynch, C.; Kramer, S.; Whiteley, G. R.; Wall, M. R.; Mannion, D. P.; Della Cioppa, G.; Rakitan, J. S.; Wolfe, G. M. High-Resolution Serum Proteomic Profiling of Alzheimer Disease Samples Reveals Disease-Specific, Carrier-Protein-Bound Mass Signatures. Clin. Chem. 2005, 51, 1946-1954.

15. Gerlich, D. Applications of RF Fields and Collision Dynamics in Atomic Mass Spectrometry. J. Anal. At. Spectrom. 2004, 19, 581-590.

16. Tanner, S. D.; Baranov, V. I.; Bandura, D. R.Reaction Cells and Collision Cells for ICP-MS: A Tutorial review. Spectrochim. Acta B57136120021452

17. Gerlich; D. Advances in Chemical Physics. LXXXII Inhomogeneous RF Fields: A Versatile Tool for the Study of Processes with Slow Ions; John Wiley and Sons: New York, 1992, pp 1-176.

18. Dahl, D. A. SIMION for the Personal Computer in Reflection. Int. J. Mass Spectrom. 2000, 200, 3-25.

19. Rao, R.; Bhutani, A. Electric Hexapoles and Octapoles with Optimized Circular Section Rods. Int. J. Mass Spectrom. 2000, 202, 31-36.

20. Douglas, D. J.; Konenkov, N. V. Influence of the Sixth and Tenth Spatial Harmonics on the Peak Shape of a Quadrupole Mass Filter with Round Rods. Rapid Commun. Mass Spectrom. 2002, 16, 1425-1431.

21. Dahl,, D.http://www.simion.com/examples/dahl_drag.prg.

22. Quarmby, S. T.; Yost, R. A. Fundamental Studies of Ion Injection and Trapping of Electrosprayed Ions on a Quadrupole Ion Trap. Int. J. Mass Spectrom. 1999, 190/191, 81-102.

23. Valentine, S. J.; Counterman, A. E.; Clemmer, D. E. A Database of 660 Peptide Ion Cross Sections: Use of Intrinsic Size Parameters for Bona Fide Predictions of Cross Sections. J. Am. Soc. Mass Spectrom. 1999, 10, 1188-1211.

24. Gillig, K. J.; Bluhm, B. K.; Russell, D. H. Ion Motion in a Fourier Transform Ion Cyclotron Resonance Wire Ion Guide Cell. Int. J. Mass Spectrom. Ion Processes 1996, 157/158, 129-147.

25. Dawson, P. H. Quadrupole Mass Spectrometry and Its Applications; American Institute of Physics Press: Woodbury, New York, 1995.

26. Hagg, C.; Szabo, I. New Ion-Optical Devices Utilizing Oscillatory Electric Fields. II. Stability of Ion Motion in a 2D Hexapole Field. Int. J. Mass Spectrom. Ion Processes 1986, 73, 237-275. 